\title{
Self-Modulation of Premotor Cortex Interhemispheric Connectivity in a Real-Time Functional Magnetic Resonance Imaging Neurofeedback Study Using an Adaptive Approach
}

\author{
João Pereira, ${ }^{1,2}$ Bruno Direito, ${ }^{1,2}$ Alexandre Sayal, ${ }^{1,2}$ Carlos Ferreira, ${ }^{1,2}$ and Miguel Castelo-Branco ${ }^{1,2}$
}

\begin{abstract}
Recent studies have reported on the feasibility of real-time functional magnetic resonance imaging (rt-fMRI) neurofeedback (NF) training. Although modulation of blood oxygenation level-dependent signal of single brain regions in rt-fMRI NF is a well established technique, the same does not hold true for modulation of connectivity. Self-modulation of interregional connectivity is a potential alternative in clinical neuroscience applications, since long-range functional dysconnectivity is being increasingly recognized as a mechanism underlying neuropsychiatric disorders. In this study, a framework was designed to train participants to self-regulate, in real time, interhemispheric functional connectivity between bilateral premotor cortices. To this end, participants use a novel adaptive motor imagery task, with gradual frequency variation preventing activity plateaus and subsequent decreases in correlation of activity (three NF runs). Participants were able to upregulate and maintain interhemispheric connectivity using such adaptive approach, as measured by correlation analysis. Modulation was achieved by simultaneous volitional control of activity in premotor areas. Activation patterns in the downregulation condition led to significantly lower correlation values than those observed in the upregulation condition, in the first two NF runs. Comparison between runs with and without feedback showed enhanced activation in key reward, executive function, and cognitive control regions, suggesting NF promotes reward and the development of goal-directed behavior. This proof-of-principle study suggests that functional connectivity feedback can be used for volitional self-modulation of neuronal connectivity. Functional connectivity-based NF could serve as a possible therapeutic tool in diseases related to the impairment of interhemispheric connectivity, particularly in the context to motor training after stroke.
\end{abstract}

Keywords: functional connectivity-based neurofeedback; interhemispheric connectivity; premotor cortex; real-time functional magnetic resonance

\section{Introduction}

$\mathbf{F}$ UnCTIONAL MAGNETIC RESONANCE IMAGING (FMRI) can be used as a noninvasive technique using the amplitude of the blood oxygenation level-dependent (BOLD) signal for neurofeedback (NF) applications (Sulzer et al., 2013). Technical and methodological advances supported the implementation of real-time fMRI (rt-fMRI) and have allowed for online brain activation monitoring and the possibility of presenting this information to the participant while he/she is performing a particular task (Sitaram et al., 2011). NF is a technique that allows participants to have real-time access to measures of their own brain activity to learn to volitionally self-modulate activity in a specific brain region, combination of regions, or even connectivity between regions. A wide range of neurological disorders have been shown to present impaired brain activation patterns, and NF-based training may represent a safe therapeutic alternative, enabling neurobehavioral improvements, with potential to help patients on a long-term basis (Coben et al., 2010).

\section{From single region of interest NF to connectivity-based NF}

Since the first rt-fMRI NF experiments (Weiskopf et al., 2004), many studies have reported single region of interest (ROI) self-modulation related to particular cognitive

\footnotetext{
${ }^{1}$ Institute for Biomedical Imaging and Life Sciences (CNC.IBILI), Faculty of Medicine, University of Coimbra, Coimbra, Portugal.

${ }^{2}$ CIBIT, Coimbra Institute for Biomedical Imaging, Institute of Nuclear Sciences Applied to Health (ICNAS), University of Coimbra, Coimbra, Portugal.
} 
functions (Caria et al., 2010; Direito et al., 2019; Rota et al., 2009; Weiskopf et al., 2004; Sitaram et al., 2017). To confirm the beneficial effects on brain function, neuroplasticity, and cognitive improvement, specific behavioral outcome measures are often used to document training effects (Weiskopf, 2012).

The fact that complex cognitive processes (emotion processing, motor coordination, language, etc.) imply coordinated activity between several brain regions has so far been overlooked in this context (Friston, 2011). Despite the positive evidence favoring the presence of beneficial effects of self-regulation of a specific brain region and induced behavioral changes, such a simplistic approach may not address the complexity of the underlying connectivity-based processing mechanisms (Ruiz et al., 2014).

Disorders such as autism (Minshew and Keller, 2010), movement disorders (Poston and Eidelberg, 2012), schizophrenia (Stephan et al., 2006), and stroke (New et al., 2015) have been associated with deficient or poorly coordinated brain networks and with the loss or destabilization of interhemispheric connectivity. In this sense, there has been an increasing number of studies analyzing connectivity across brain networks, based on bivariate or multivariate covariance or synchronization of segregated brain areas (Bullmore, 2012).

Recent developments in NF experiments focused on the activation of more than one brain region. Koush et al. (2013) tested the feasibility of using effective connectivity measures in a NF setup, in which the participants were asked to modulate connectivity between ipsilateral brain regions from visual and parietal cortices. The feedback display was designed based on a "near" real-time implementation of dynamic causal modeling. The feedback was computed using a 90-point sliding window and visually presented after $60 \mathrm{sec}$. Ruiz et al. (2014) studied an rt-fMRI NF setup based on functional connectivity metrics. The authors proposed healthy participants to increase functional connectivity between the inferior frontal gyrus (IFG) and the superior temporal gyrus, using visual feedback. The feedback was calculated as a sliding window correlation coefficient considering current and past time points. Spetter et al. (2017) developed a functional connectivity-based rt-fMRI NF experiment, in which subjects were instructed to increase functional connectivity between the dorsolateral prefrontal cortex (dlPFC) and the ventromedial prefrontal cortex, key areas in executive control and reward processing. A similar approach was used for patients with chronic stroke and severe motor impairment with reported augmentation in ipsilesional cortical-subcortical connectivity (Liew et al., 2016).

\section{Motor imagery paradigm}

Motor imagery (MI) is defined as a dynamic state in which the subject mentally simulates a particular action so that he/ she can feel himself as if performing the imagined action (Decety, 1996). Compared with the brain processes involved in motor performance (MP), MI requires an additional inhibitory process from prefrontal and dorsolateral frontal cortices to prevent the imagined movement from actually happening (Jeannerod, 1994; Piokenhain, 1984).

Functional imaging studies (Gao et al., 2011; Gerardin et al., 2000) have shown an overlap between the neural networks involved in MI and MP, reporting coincident activations in several areas such as the supplementary motor area
(SMA), premotor cortex (PMC), parietal areas, the basal ganglia, and the cerebellum. Guillot et al. (2014) reported higher activity in the ventral part of the PMC, responsible for movement planning during MI.

Interhemispheric functional interactions between motorrelated areas in both MI and MP tasks have been previously reported (see the studies by Gao et al., 2011, Grefkes et al., 2008, and Liuzzi et al., 2011) and their relevance in terms of movement coordination was highlighed.

There is an important distinction in the MI literature regarding visual and kinesthetic MI and related brain activation patterns (Andrade et al., 2017; Hétu et al., 2013).

In particular, kinesthetic MI (a first-person perspective) has been previously used in reference tasks for NF experiments (Berman et al., 2012; Linden and Turner, 2016; Mehler et al., 2019). Authors reported high correlation between self-modulation of motor areas and improvements in taskrelated MP (Blefari et al., 2015).

In this proof-of-concept experiment, we asked participants to voluntarily modulate the interhemispheric connectivity between bilateral premotor cortices. The Pearson's correlation coefficient was used as the connectivity measure between the BOLD signals of these two regions.

Taking into account the novelty of this technique, we tested the ability to up- and downregulate functional connectivity. The feedback was presented continuously during NF functional runs.

We hypothesized that (1) it is possible to modulate interhemispheric connectivity between right- and left-hemisphere PMC in an rt-fMRI NF experiment based on a kinesthetic MI paradigm and (2) participants are able to adaptively optimize (based on imagined frequency of movement) the modulation strategy using such an NF paradigm.

\section{Materials and Methods}

\section{Participants}

We recruited 10 volunteers (mean age: $26.5 \pm 3.3 ; 7$ male) to perform a functional connectivity-based rt-fMRI NF experiment. This sample size was decided based on similar proof-of-concept studies (Thibault et al., 2018). All had normal or corrected-to-normal vision and no history of neurological or psychiatric diseases. All volunteers gave informed consent before participating. This study was conducted in accordance with the declaration of Helsinki.

\section{Pretraining and psychometric evaluation}

The NF sessions were preceded by a 30-min training session in which each participant got acquainted with the fMRI protocol and reference tasks. All questions concerning the study were answered. Their MI ability was assessed using the Movement Imagery Questionnaire-3 (Mendes et al., 2016; see Results in Supplementary Table S1).

After the fMRI session, all participants answered a debriefing questionnaire, in which they reported their subjective experience and perceived success of the proposed adaptive strategies.

\section{rt-fMRI NF data acquisition}

Each rt-fMRI NF session consisted of a first anatomical run-a run where a structural information of the brain is 
acquired-followed by six functional runs. Functional runs are defined as task-related runs where BOLD signals are measured: the first includes a localizer run that is used to map PMC. This run defines the spatial mask used as a NF target region. The localizer run is followed by five imagery runs. The first and the last imagery runs were performed without feedback information. Each scanning session lasted for $\sim 1 \mathrm{~h}$ and $20 \mathrm{~min}$.

The setup includes a 3T Siemens Magnetom TrioTim scanner with a 12-channel head coil. First, the participants underwent a high-resolution magnetization-prepared rapid acquisition gradient echo sequence for coregistration of functional data (176 slices; echo time [TE]: $3.42 \mathrm{~ms}$; repetition time [TR]: $2530 \mathrm{~ms}$; voxel size: $1 \times 1 \times 1 \mathrm{~mm}$; flip angle [FA]: $7^{\circ}$; field of view [FOV]: $256 \times 256 \mathrm{~mm}$ ).

Functional imaging focused on motor-related areas (related to motor planning and/or execution in frontal and parietal areas). The acquisition consisted of an echo-planar imaging sequence with 26 slices, in-plane resolution: $3 \times 3 \mathrm{~mm}$, FOV: $210 \times 210 \mathrm{~mm}$, slice thickness: $3.5 \mathrm{~mm}$, FA: $75^{\circ}, \mathrm{TR}=1500 \mathrm{~ms}$ and $\mathrm{TE}=30 \mathrm{~ms}$. The real-time data processing methods required to compute and present the feedback were performed using Turbo-BrainVoyager 3.2 (TBV; Brain Innovation, Maastricht, The Netherlands). The software implements real-time three-dimensional head motion detection and correction as well as an alignment between functional runs using anatomical information as reference. An incremental general linear model (GLM) based on a recursive squares algorithm accessed the functional data as soon as available.

Stimuli were presented on an LCD screen $(70 \times 39.5 \mathrm{~cm}$, resolution of $1920 \times 1080$ pixels, $60 \mathrm{~Hz}$ refresh rate) that the participants viewed through a mirror mounted above their eyes at an effective distance of $156 \mathrm{~cm}$.

\section{Functional ROIs definition}

To functionally localize the NF target regions during the localizer run, we used a mapping procedure that combined movement imagination with actual performance, following the task proposed in Xie et al. (2015). The procedure included two activation conditions, MI and MP, and baseline periods in a block design experiment.

The mapping procedure included a total of 17 blocks of 30 sec- 9 baseline blocks, 4 MI blocks, and 4 MP blocks. The total duration of the localizer was $8 \mathrm{~min}$ and $30 \mathrm{sec}$. At the beginning of each block, the condition name was presented to the participant on the screen for $3 \mathrm{sec}$. During the MP blocks, each participant was instructed to execute a bilateral finger tapping sequence: 1-2-1-4-3-4 (1-left middle finger, 2-left index, 3-right index, and 4-right middle finger) at a specific frequency $(2 \mathrm{~Hz})$. During the MI blocks, the participants were asked to imagine the same sequence, without overt movement.

The ROIs (right premotor cortex [rPMC] and left premotor cortex $[\mathrm{lPMC}])$ were functionally defined according to the contrast $\mathrm{MI}>$ baseline. In addition, anatomical landmarksanterior to the central sulcus and superior to the Sylvian fissure, caudal parts of the superior frontal gyrus and middle frontal gyrus - and functional information - the rostral part of the precentral gyrus not occupied by the primary motor cortex (M1; Schubotz et al., 2010)—were used to determine the appropriate ROIs. The contrast MP > baseline was used to validate the strategy.

\section{Feedback calculation and stimulus presentation}

During the NF runs, the mean BOLD values of each ROI (defined in the functional localizer, rPMC and IPMC) were used to compute the functional connectivity metric according to Equation 1:

$$
p=\frac{\sum_{i=1}^{n}\left(x_{i}-\bar{x}\right)\left(y_{i}-\bar{y}\right)}{\sqrt{\sum_{i=1}^{n}\left(x_{i}-\bar{x}\right)^{2} \cdot\left(y_{i}-\bar{y}\right)^{2}}}=\frac{\operatorname{cov}(X, Y)}{\sqrt{\operatorname{var}(X) \cdot \operatorname{var}(Y)}},
$$

where $p$ represents Pearson's correlation coefficient, $x$ and $y$ the mean BOLD signal of each ROIs, and $n$ the sliding window size (Zilverstand et al., 2014). Feedback was calculated using an eight-point window (12 sec interval) and was updated at every point $(1.5 \mathrm{sec})$.

A three-sample smoothing was applied before displaying the feedback value to avoid sudden variations (Zhang, et al., 2007) and to minimize frustration and fatigue of the participants.

The visual feedback was presented through a thermometer bar divided into 20 blocks, each block representing a 0.1 correlation increment ( $p$ values range from -1 to +1 ). TBV was used to extract the mean BOLD time courses and a custom Matlab software (The Mathworks, Natick, MA) script was developed to calculate the correlation and present the visual feedback (Fig. 1).

The imagery runs were composed of 17 blocks of $30 \mathrm{sec}$, totaling $510 \mathrm{sec}$ (340 volumes). The first and the last imagery runs were performed without feedback to provide a withinsubject control strategy and test the ability to control BOLD connectivity in the absence of the feedback information. An auditory beep at the $15 \mathrm{sec}$ mark indicated the midpoint for each block.

\section{NF proposed strategies}

The imagery runs included two conditions: "Increase", and "Decrease." Each condition was presented eight times per run, alternately. During the "Increase", blocks, the participants were asked to implement an adaptive strategy to fill up the thermometer bar. On the opposite, during the "Decrease" blocks, they were instructed to implement a strategy that would keep the thermometer as close to zero as possible. For both conditions, negative values in the thermometer were to be interpreted as negative feedback.

Based on data collected during pilot experiments, the adaptive strategy proposed to increase the thermometer value ("Increase" condition) consisted of the kinesthetic imagery of bimanual synchronized movements with gradual increase in frequency until the $15 \mathrm{sec}$ mark, followed by a gradual decrease in the frequency until the end of the block. The critical rationale of this strategy is to avoid activity "plateaus" where correlations become nondiscernible, and to promote activity variations that favor detection of correlation patterns. Our pilot data favored this hypothesis, and are further explained in Figure 2-a triangular shape time course of the mean BOLD value of both rPMC and IPMC would increase the likelihood of identifying functional correlations. The adaptive strategy proposed is also in accordance 


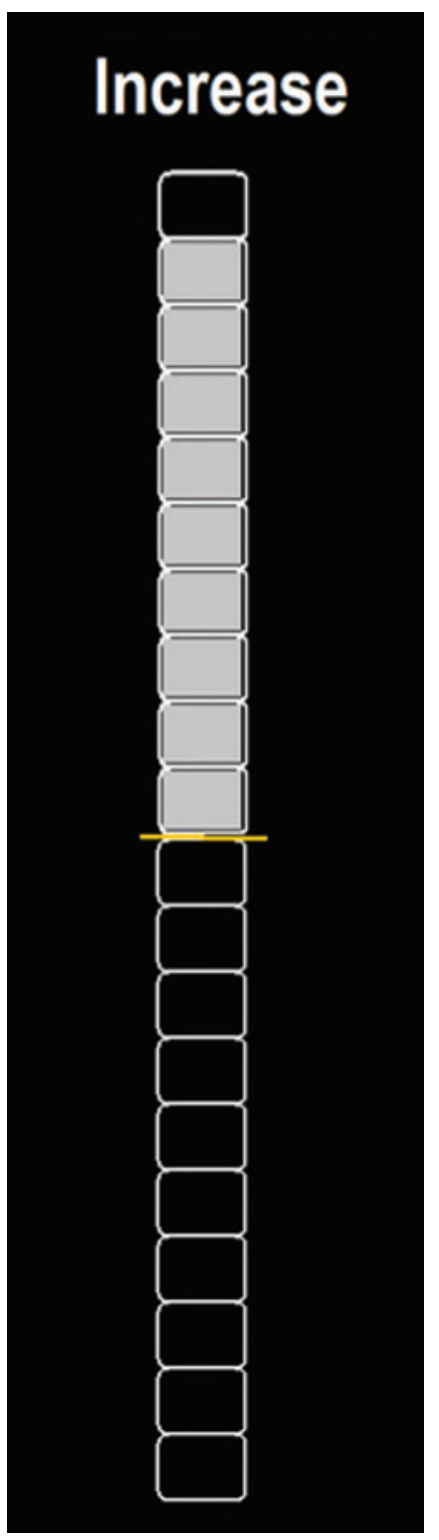

FIG. 1. Visual feedback and presented instruction (with the name of the condition ("Increase" or "Decrease") to each participant, at the beginning of each block, during the $\mathrm{NF}$ runs. NF, neurofeedback. Color images are available online.

with Rao et al. (1996), that is, the activation level within the whole-motor network increases with increasing finger tapping speed, that is, frequency increase.

For the "Decrease" condition block, participants were instructed to use a mental strategy of their own choice that did not activate premotor areas.

\section{Offline fMRI data analysis}

Offline fMRI data analysis was performed using BrainVoyager QX 2.8 (Brain Innovation). Preprocessing included slicetime correction, motion detection and correction, temporal high-pass filtering, coregistration between the functional and the anatomical data, and normalization to Talairach (TAL) coordinate space (Talairach and Tournoux, 1988).

\section{Whole-brain statistical analyses}

Functional data from the localizer and imagery runs were analyzed using a GLM. The design matrix was determined based on the predictors encoding the stimulus conditions, combined with six motion parameters (three translational and three rotational) and spikes (i.e., outliers in the BOLD time course), which were included as covariates.

A first whole-brain exploratory fixed effects (FFX-GLM) group analysis was performed to determine differences in the magnitude of the BOLD signal between conditions during the functional runs. During the localizer run, we aimed to determine the optimal regions for NF targets. During imagery runs, the objective of the whole-brain analysis was to explore the most relevant regions involved in the presence or absence of feedback and explore the underlying neural mechanisms involved in the proposed imagery tasks.

\section{Connectivity analysis}

Functional connectivity was determined as the Pearson's correlation between mean BOLD time courses of the NF targets (rPMC and IPMC).

To evaluate the ability to self-regulate connectivity in the NF runs, we compared the correlation values of both conditions ("Increase" vs. "Decrease") at the midpoint of each block (i.e., TR sample 10, where an auditory cue instructs a decrease in imagined frequency). According to the triangular shape activation pattern hypothesis, this timepoint is crucial to understand whether this optimized strategy would result in a better differentiation of correlation between conditions. The selection of a time point well within the condition block also ensures that the feedback is entirely resulting of mental strategies of the current condition (taking into account the inherent BOLD signal delay and the windowed correlation calculation).

To further explore the modulation of functional connectivity, we compared the Pearson's correlation values of the condition "Increase" versus condition "Decrease." To this end, only the final 12 correlation values from each block were considered (the rationale is that the first 8 points represent a mixture of data between both conditions-preventing points from the previous block from a different condition from being considered).

A paired Wilcoxon sign rank test was performed to determine the statistical significance of the difference between the conditions across participants and runs.

\section{Results}

\section{Localizer run and NF target}

The localizer run allowed for online functional definition of the subject-specific NF targets with the mapping procedure depicted in Figure 3.

For each subject, significant activation clusters were selected for IPMC and rPMC ROIs. Mean location of the ROIs were IPMC: $(-48,-4,44)$ TAL coordinates, with size $819 \pm 503 \mathrm{~mm}^{3}$ and rPMC: $(46,-6.5,48)$, with size $636 \pm 334.97 \mathrm{~mm}^{3}$. The probabilistic maps corresponding to the overlap of the subject-specific ROIs defined online are presented in Figure 4.

\section{BOLD modulation patterns}

A block-related average (20 TRs corresponding to the $30 \mathrm{sec}$ condition block) of the mean BOLD time course 

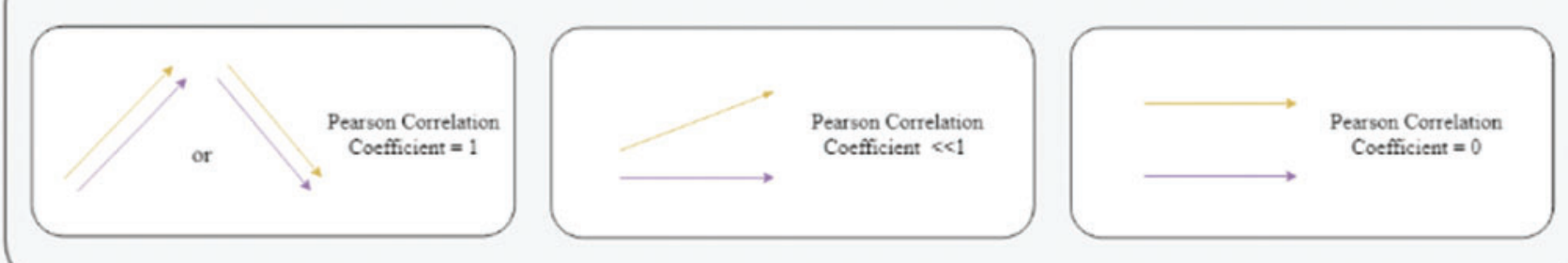

B Expected results when applying to fMRI data

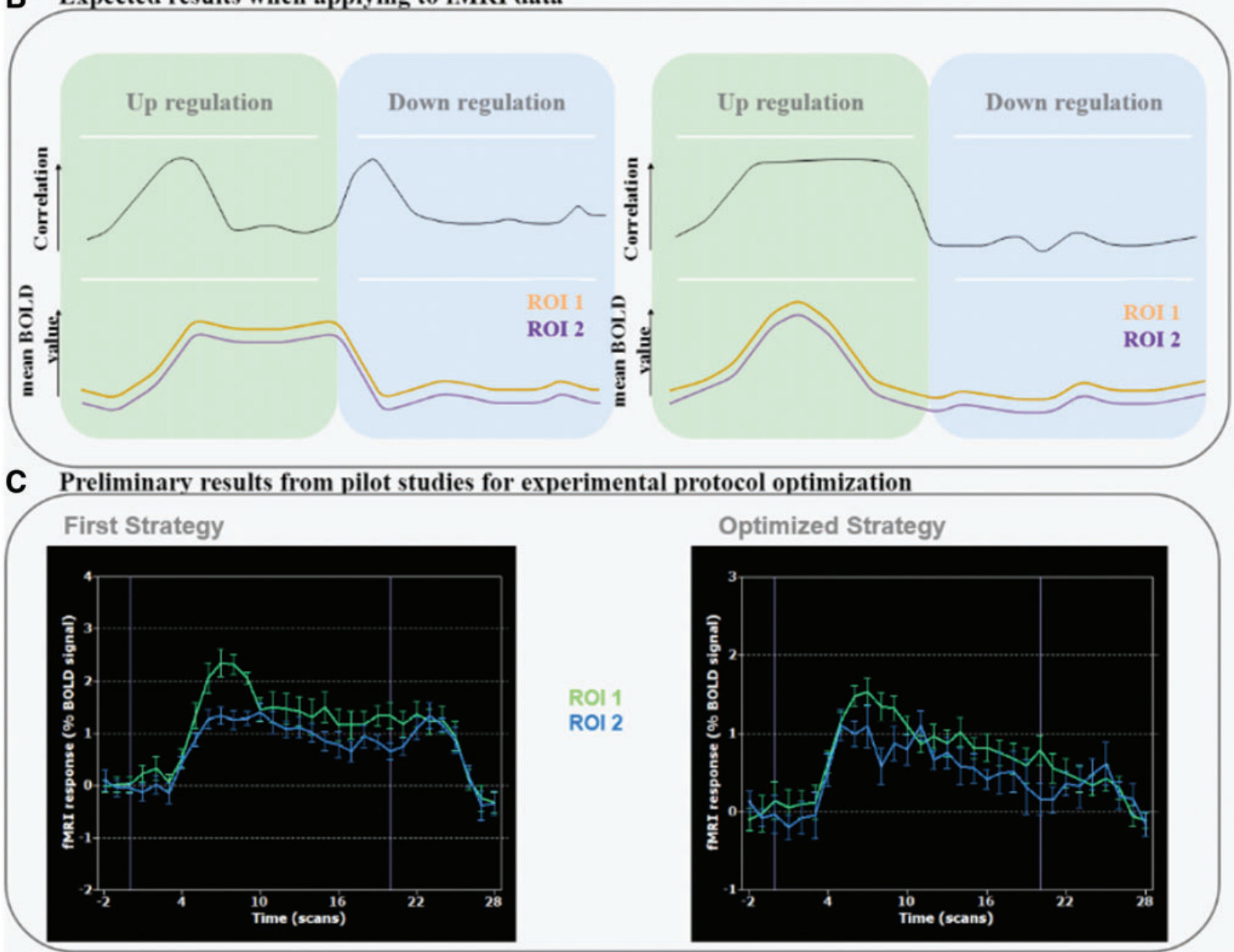

FIG. 2. (A) Theoretical model of the Pearson's correlation coefficent as a functional connectivity measure with the arrows indicating synchronized activation and deactivation of two ROIs as both resulting in a nearly perfect correlation. (B) Schematic representation of the theoretical behaviour of mean BOLD signal and predicted consequent correlation values for two distinct approaches during the "upregulation" condition: using a simple activation strategy for both premotor areas, resulting in a "plateau" in mean BOLD values after an initial peak with its decrease occurring already within the next downregulation condition (left) and using a gradual frequency variation in the activation strategy, resulting in a triangular shape time course of the mean BOLD value of both ROIs and allowing for the BOLD signal to be low in the beginning of the next condition (right). (C) Preliminary results obtained during a pilot study phase for experimental protocol optimization, with the variation of the fMRI response (\% BOLD signal) using the first mental strategy (left) and using the second mental strategy (right) in bilateral premotor areas (ROI 1 and ROI 2). BOLD, blood oxygenation level-dependent; fMRI, functional magnetic resonance imaging; MRI, magnetic resonance imaging; ROI, region of interest. Color images are available online. 

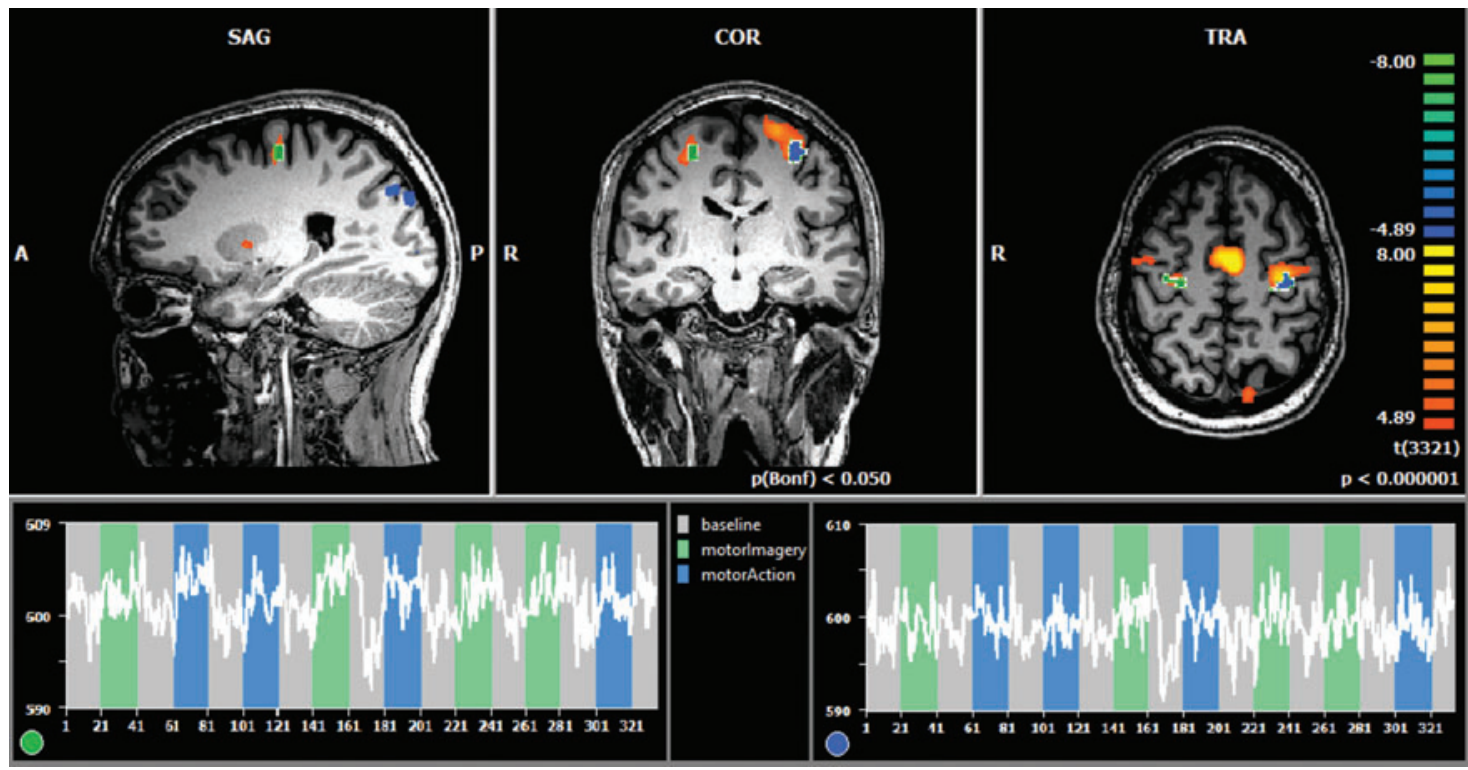

FIG. 3. Overview of the localizer run, with offline FFX group analysis, $p<0.05$ (Bonferroni corrected), overlaid with the online selected ROIs (blue, IPMC; green, rPMC) of one chosen subject on top and their respective BOLD signal time course (gray blocks: "baseline" condition, green blocks: "motor imagery" condition, blue blocks: "motor performance" condition) on the bottom. FFX, fixed effects; IPMC, left premotor cortex; rPMC, right premotor cortex. Color images are available online.

considering all NF runs (10 subjects, 3 runs each) for each ROI is presented in Figure 5. The average time course depicted in the figure for the "Increase" condition (green) shows a bilateral increase of the mean BOLD during the first samples, as hypothesized. This pattern is followed by a gradual decrease toward the end of the block. At the beginning of "Decrease" blocks, the mean BOLD activity decreases bilaterally.

The prominent shape of BOLD signal time course for both ROIs in the "Increase" condition departs from a "plateau" and follows a triangular (increasing, peaking, and decreasing) shape, suggesting that the adaptive strategy was successful.

\section{Functional connectivity during imagery runs}

Midpoint of upregulation block: difference between conditions. To explore possible modulation of functional con- nectivity between bilateral premotor areas, we analyzed the differences between correlation values for each condition at the midpoint of the blocks (corresponding to the expected "triangle peak"), that is, volume 10 .

The difference between the functional connectivity at this critical midpoint is statistically significant in NF runs 1 $(Z=3.377, p<0.001)$ and $2(Z=3.736, p<0.001)$. The participants were unable to achieve statistically different correlation values between conditions in the very last run 3 $(Z=0.475, p=0.635)$.

As mentioned in the section "rt-fMRI NF data acquisition," before and after the NF runs, functional runs without NF were also acquired. Regarding those runs, statistical significance was observed in the "before" run but not in the "after" run [Z("before") $=3.799, p<0.001 ; Z$ ("after") $=1.118, p=0.264]$. All functional runs presented upregulation average values greater than the downregulation average values.
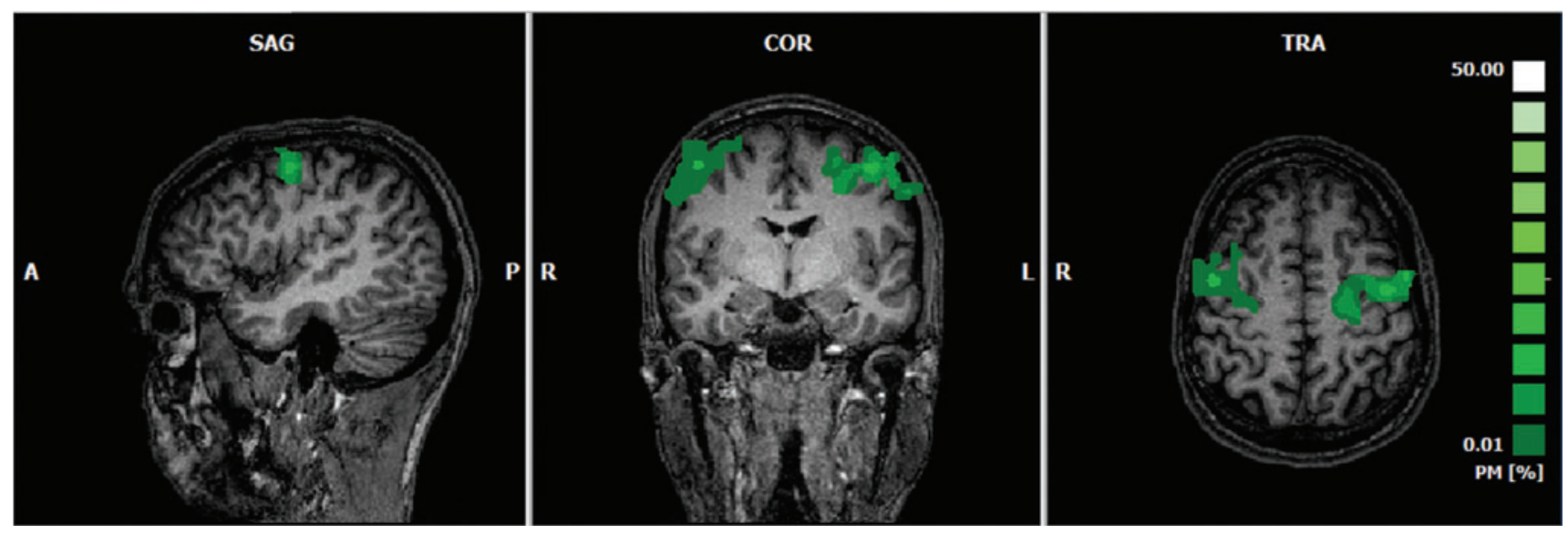

FIG. 4. Probabilistic map of the combined ROIs (IPMC and rPMC) for the 10 subjects, showing the overlap of the subjectspecific ROIs, selected online during the localizer run. Color images are available online. 


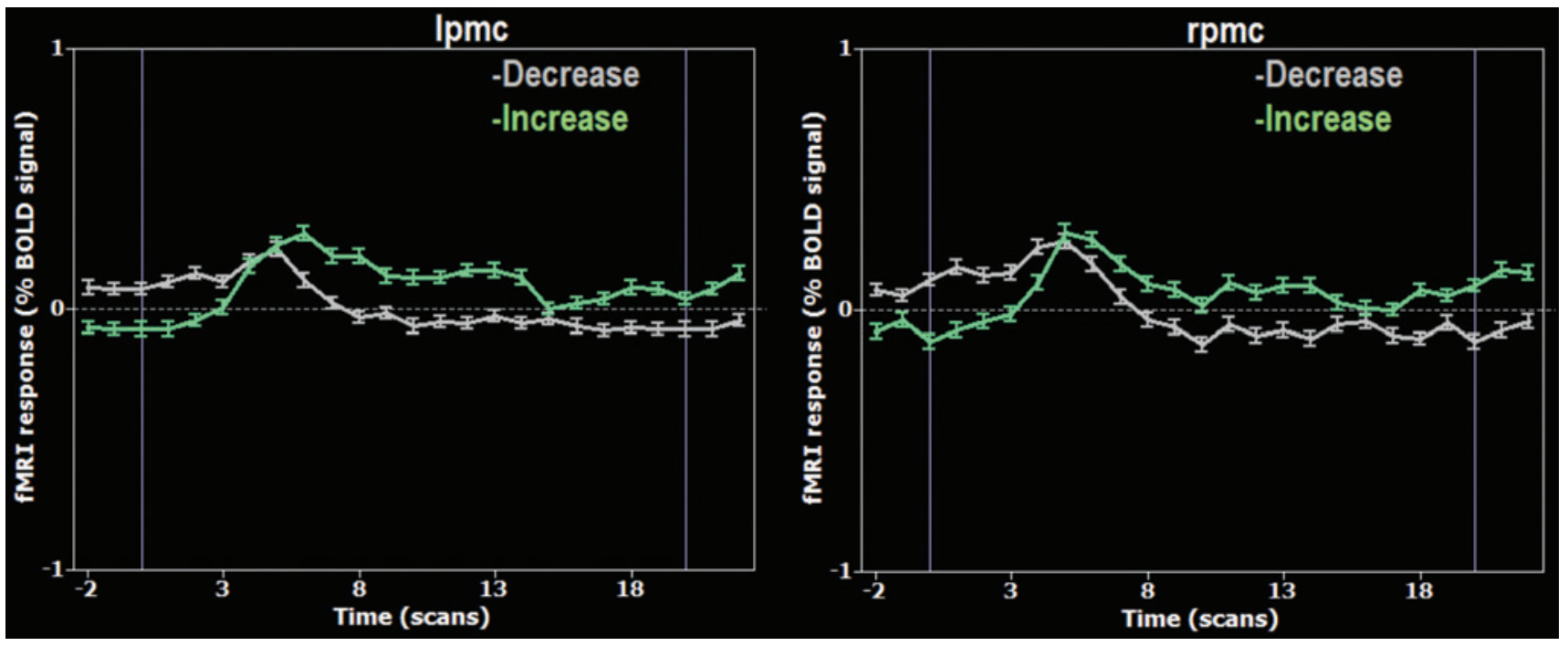

FIG. 5. Block-related average per time point (20 TRs) of the mean BOLD time course of the lPMC (left) and rPMC (right), for both "Increase" (green) and "Decrease" (gray) conditions, for all 10 subjects and the three NF runs. TR, repetition time. Color images are available online.

Figure 6 illustrates the differences (box-plots with confidence intervals) between the functional connectivity at midpoint (volume 10) across all NF runs and subjects. The results show a significant difference between "Increase" and "Decrease", correlation values $(Z=4.177, p<0.001$, paired tests).

Whole-block connectivity analysis. Regarding the comparative analysis between conditions based on the 12-point window, participants presented higher functional connectivity during the condition "Increase" in NF runs 1 (marginally) and 2 (highly significant) $[Z(N F 1)=1.833, p=0.067$ and $Z(N F 2)=4.501, p<0.001]$. On the opposite, the "Decrease", condition presented higher functional connectivity in run 3 $[Z(N F 3)=-7.182, p<0.001]$. This intriguing finding may be explained by decay of attention in this late run, as suggested by debriefing (see Discussion section). In the runs without feedback, functional connectivity modulation did not change across conditions $[Z($ "before") $=0.564$, $p=0.573 ; Z$ ("'after") $=-1.098, p=0.272]$.

\section{Statistical maps}

NF runs. Whole-brain analysis contrasting "Increase"> "Decrease" conditions presented activation clusters in bilateral precentral gyrus (corresponding to the premotor areas), medial frontal gyrus (MFG; corresponding to the SMA), bilateral IFG (related with executive function), bilateral lentiform nucleus, and bilateral anterior lobe of the cerebellum (both related to motor control; Fig. 7).

Feedback versus nonfeedback. To study the effect of feedback in the performance of the imagery task, we analyzed activation patterns of the brain in runs in which feedback was given and runs in which feedback was not (Fig. 8). Considering our contrast of interest ("Increase") "Decrease"), the difference between feedback and nonfeedback runs showed activation clusters in areas such as MFG, anterior cingulate cortex, middle occipital gyrus, parahippocampal gyrus, middle temporal gyrus, fusiform gyrus, and areas of the midbrain such as the thalamus.

\section{Discussion}

The main achievement of this article was the development of a NF training technique that allows to modulate functional connectivity and to maintain this modulation consistently throughout an experimental block. This study hence addresses an important research gap in the development of connectivity-based NF paradigms. Specifically, the typical BOLD signal response of a block design paradigm task tends to "plateau" after some time, leading to lower correlation values. This is a major challenge for the use of correlation as a NF metric. By instructing a strategy of gradual variation of movement imagery frequency (increasing up to the midpoint of the block followed by a gradual decrease), we prevented such "plateau" thereby enabling participant's stable modulation of the correlation.

With this novel strategy, modulation of functional connectivity was achieved due to simultaneous self-modulation of bilateral premotor activity by increases and decreases of BOLD response. The triangular-shaped BOLD signal pattern prevented the emergence of a "plateau" during the upregulation condition as hypothesized. Moreover, lower connectivity values were observed in the "Decrease" condition than in the "Increase" condition. However, the concomitant bilateral decrease of activity in the early time points of the "Decrease" condition may lead to an initial and undesired increase in correlation.

Group-level analysis exhibits significant differences while comparing critical midpoint correlation values from upregulation and downregulation conditions in the first two of the three NF runs. In addition, the analysis based on a 12 points window of each block supports the idea of maintenance of higher values of correlation during upregulation condition comparing with downregulation condition. The results of 

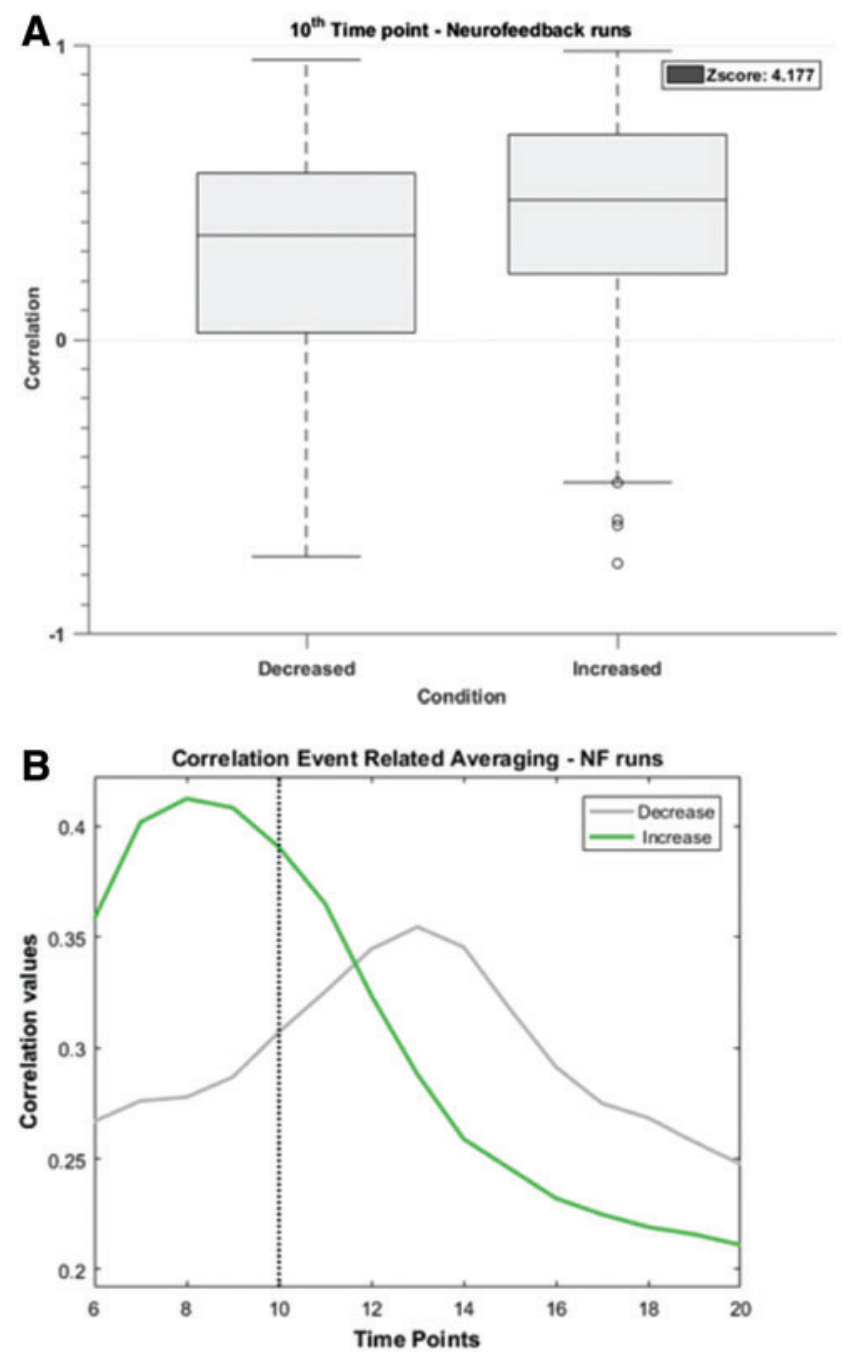

FIG. 6. (A) Differences between the functional connectivity in "Increase" and "Decrease" conditions at TR 10 for all NF runs across all subjects with the $z$-score of paired Wilcoxon test. (B) Block-related average of the correlation values for each time point for both "Increase" (green) and "Decrease" (gray) conditions, for all 10 subjects and the three NF runs. The vertical line at the 10th time point represents the TR used for calculation. Color images are available online.

the last NF run, as well as the results of the last functional run ("after") could possibly be due, as suggested by debriefing, to raised questions by the participants of attention span and exhaustion, especially in the latter stages of the session. These factors were already reported in previous NF experiments (Greer et al., 2014; Koush et al., 2013) and could likely contribute to the correlated late decay of the BOLD signal to a lower level of activity in the NF targets BOLD signal during the "Decrease" condition.

Previous studies have shown that the modulation of connectivity between different regions is possible (e.g., dlPFC-amygdala regions, Zotev et al., 2013), but to the best of our knowledge, this is the first study aiming interhemispheric connectivity modulation and proposing/assessing specific strategies to stably modulate (increase or decrease) connectivity.
The results suggest that the presence of feedback had an impact on the ability of the participants to modulate functional connectivity. The control runs, without feedback, presented nonsignificant or smaller differences between "Decrease" and "Increase" conditions than the differences achieved in the NF runs, suggesting that the presence of NF is instrumental: participants relied on the feedback information presented to adapt their brain response and update the strategy used.

We were also interested in the direct neural correlates of feedback presentation. To this end, we investigated which regions presented differences between the feedback and the nonfeedback runs. Regions such as MFG and caudate, key areas related to executive functions, cognitive control, and the reward-related system (Ridderinkhof et al., 2004) as well as areas related to motor control presented higher activation during runs with feedback. The involvement of reward-related areas suggests that the participants use feedback to achieve reward, promoting the development of goal-directed behavior.

Choosing an efficient mental imagery strategy to achieve volitional control and maintenance of brain connectivity is one of the challenges in NF training. Previous studies evidenced that explicit strategies could have a positive effect on NF performance (Sulzer et al., 2013). In this study, we encouraged participants to modulate activity in two different conditions, alternating up- and downregulation of connectivity. In the first ("Increase" condition), based on the basic neurophysiological findings of Rao et al. (1996), we targeted a triangular shaped activation function (Fig. 2). To this end, we added an auditory cue indicating the midpoint of the "Increase" condition blocks. The first section of the proposed triangle-shaped activation function was achieved. These results support our hypothesis that an instructed gradual increase/decrease of BOLD signal, based on auditory midpoint cues within a condition block paradigm, is suitable for rt-fMRI functional connectivity-based NF experiments. The difficulty in sustaining high correlation values during the entire block was still present. Although highlighting the fact that this is a single-session study, the results reinforce that there is still room to improve the strategy, in multiple session paradigms.

Using the explicit frequency-based adjustment strategy proposed, the participants were able to fine-tune the kinesthetic imagery experience based on the received feedback. In the debriefing questionnaire, for the "Increase" condition, participants reported achieving best results with bimanual symmetric arm movement (playing musical instruments, e.g., drums or piano), opening and closing both hands, finger tapping sequences, and clapping. For the "Decrease" condition, in which the participants were asked to keep the thermometer as close to zero as possible, strategies included distracting mathematical calculation, N-back tasks, and color imagery.

The main limitations of this study include the relatively small sample size of healthy participants, therefore, lacking evaluation in a clinical population, the single-session approach (precluding the possibility to study learning across sessions) and the absence of electromyography recordings. A point to address in the future is to take into account situations wherein connectivity increases upon concomitant deactivation. With this design, we did not control for activation/ 

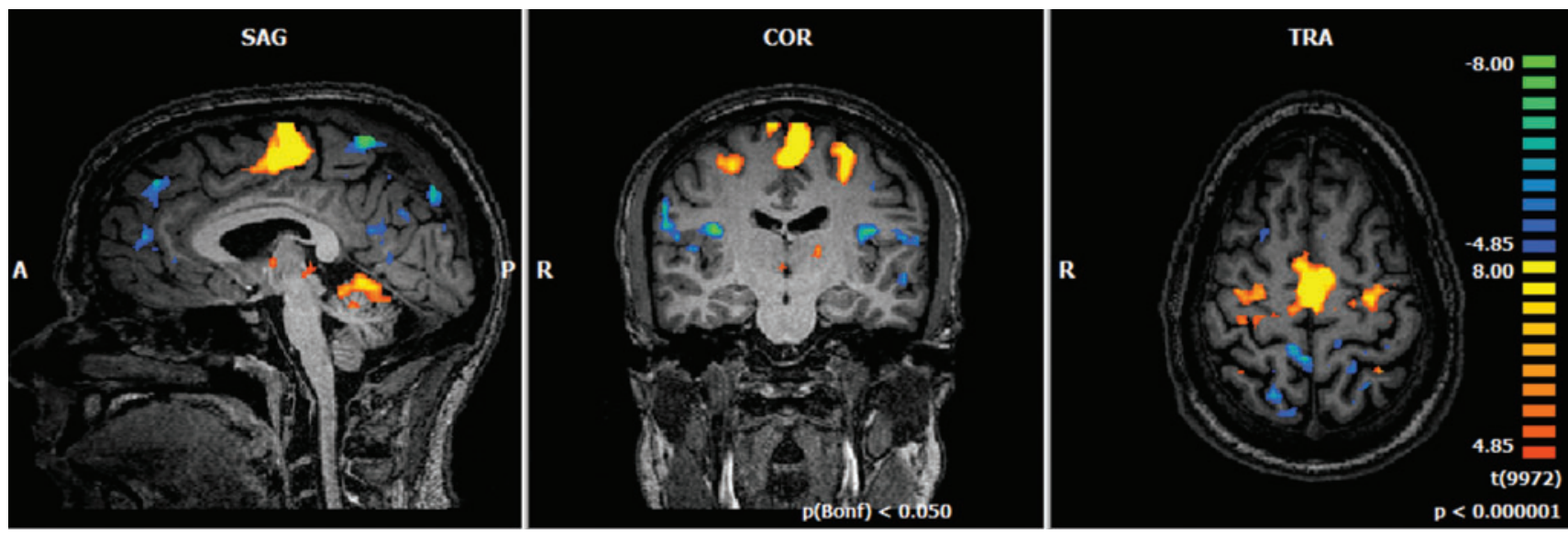

FIG. 7. FFX-GLM group analysis for all 10 subjects and all 3 NF runs per subject, with condition "Increase" greater than "Decrease," $p$ (Bonf) <0.05. GLM, general linear model. Color images are available online.

deactivation of the target regions, in the sense that both can lead to positive correlations. We focused instead on the maintenance of modulation of functional connectivity, which is achievable for both synchronous activation and deactivation of the regions of interest. The question of the directionality of the activity in target areas is also relevant regarding neurorehabilitation and should be covered in future studies. Adressing and mitigating factors such as fatigue, reported by the subjects, will also need to be considered in future studies as well as finding an optimized strategy for the "downregulation" condition. The initial transient block change caused initial relatively high correlation values in downregulation conditions (because concomitant decay leads to increased correlation), which needs to be addressed in future studies, possibly with further experimental optimization with stricter modulation tasks/strategies and type of feedback representation.

\section{Conclusion}

This proof-of-principle study suggests that functional connectivity feedback based on bilateral premotor regions can be used to voluntarily modulate and maintain neuronal con- nectivity. Our adaptive frequency strategy allowed to achieve increased stability of functional connectivity, preventing a plateau of measured activity and, therefore, a decay in correlation. The ability to modulate connectivity was achieved due to simultaneous volitional amplitude control of BOLD response in bilateral premotor areas. Ultimately, our connectivity-based approach could be an alternative and/or complement of amplitude modulation NF.

The strategy suggested to increase the connectivity during a certain period was successful but there is still room to improve the approach to sustain the modulation for longer periods of time/sessions. Moreover, we found that the presence of feedback contributed to augment this modulation (higher correlation values correspond to upregulation condition blocks). Importantly, reward-related areas were more activated in the presence of feedback.

Our findings show that interhemispheric connectivitybased rt-fMRI NF is possible and may represent a possible therapeutic tool in diseases involving impaired interhemispheric connectivity, such as stroke. Clinical trials may represent an important next step toward establishing NF as an alternative treatment approach.
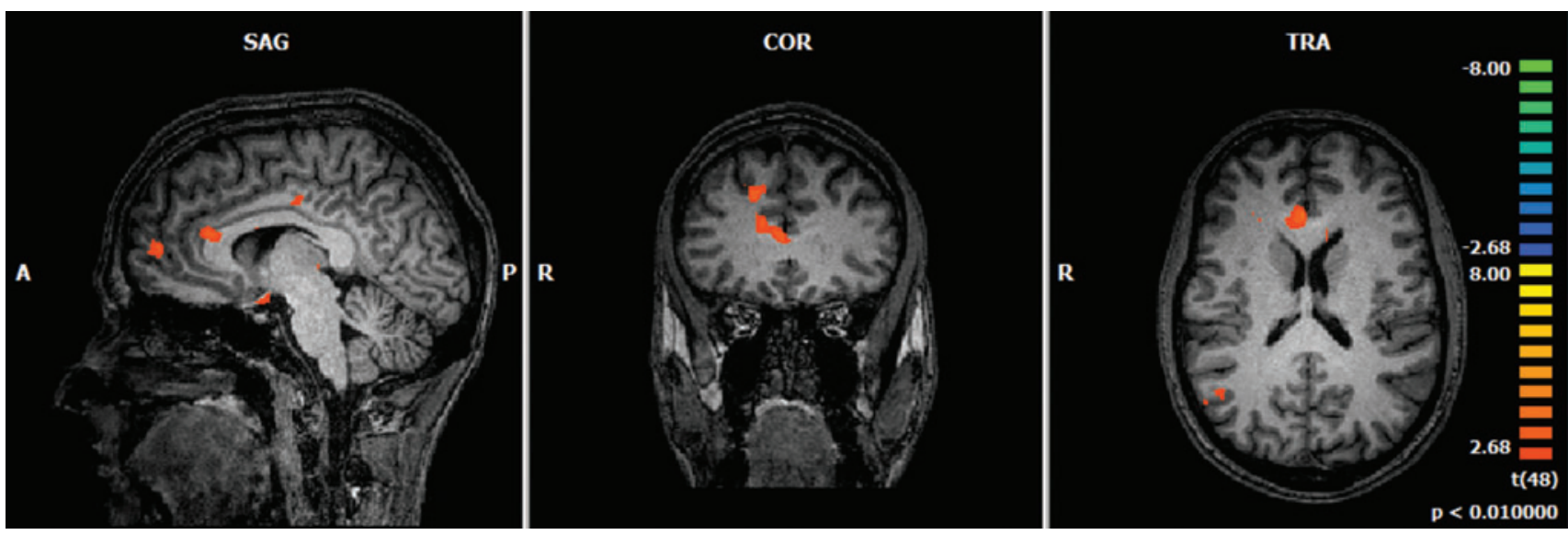

FIG. 8. Whole-brain group analysis of the difference between NF runs and nonfeedback runs, both regarding "Increase"> "Decrease" contrast, result of a statistical two-sample $t$-test comparing the activation pattern found in these two run groups, $t(48), p<0.01)$. Color images are available online. 


\section{Acknowledgments}

We thank Daniela Pereira and João Duarte for helpful suggestions.

\section{Author Disclosure Statement}

No competing financial interests exist.

\section{Funding Information}

Funding sources: FP7-HEALTH-2013-INNOVATION-1602186, BRAINTRAIN; H2020 Marie Skłodowska-Curie Actions Award Number: 708492, Fundação Luso-Americana para o Desenvolvimento, Prémio FLAD Life Sciences 2020, Fundação para a Ciência e a Tecnologia, Award Numbers: FCT-UID/NEU/04539/2013, COMPETE, POCI-01-0145FEDER-007440, POCI-01-0145-FEDER-30852, FEDER, BIGDATIMAGE, CENTRO-01-0145-FEDER-000016 financed by Centro 2020 FEDER, COMPETE; PACMEDPERSYST POCI-01-0145-FEDER-016428.

\section{Supplementary Data}

Supplementary Table S1

\section{References}

Andrade J, Cecílio J, Simões M, Sales F, Castelo-Branco M. 2017. Separability of motor imagery of the self from interpretation of motor intentions of others at the single trial level: an EEG study. J Neuroeng Rehabil 14:63.

Berman BD, Horovitz SG, Venkataraman G, Hallett M. 2012. Self-modulation of primary motor cortex activity with motor and motor imagery tasks using real-time fMRI-based neurofeedback. Neuroimage 59:917-925.

Blefari ML, Sulzer J, Hepp-Reymond M-C, Kollias S, Gassert R. 2015. Improvement in precision grip force control with selfmodulation of primary motor cortex during motor imagery. Front Behav Neurosci 9:18.

Bullmore E. 2012. The future of functional MRI in clinical medicine. Neuroimage 62:1267-1271.

Caria A, Sitaram R, Veit R, Begliomini C, Birbaumer N. 2010. Volitional control of anterior insula activity modulates the response to aversive stimuli. A real-time functional magnetic resonance imaging study. Biol Psychiatry 68:425-432.

Coben R, Linden M, Myers TE. 2010. Neurofeedback for autistic spectrum disorder: a review of the literature. Appl Psychophysiol Biofeedback 35:83-105.

Decety J. 1996. The neurophysiological basis of motor imagery. Behav Brain Res 77:45-52.

Direito B, Lima J, Simões M, Sayal A, Sousa T, Lührs M, et al. 2019. Targeting dynamic facial processing mechanisms in superior temporal sulcus using a novel fMRI neurofeedback target. Neuroscience 406:97-108.

Friston KJ. 2011. Functional and effective connectivity: a review. Brain Connect 1:13-36.

Gao Q, Duan X, Chen H. 2011. Evaluation of effective connectivity of motor areas during motor imagery and execution using conditional granger causality. Neuroimage 54:1280-1288.

Gerardin E, Sirigu A, Lehéricy S, Poline JB, Gaymard B, Marsault C, et al. 2000. Partially overlapping neural networks for real and imagined hand movements. Cereb Cortex 10:1093-1104.

Grefkes C, Eickhoff SB, Nowak DA, Dafotakis M, Fink GR. 2008. Dynamic intra- and interhemispheric interactions dur- ing unilateral and bilateral hand movements assessed with fMRI and DCM. Neuroimage 41:1382-1394.

Guillot A, Di Rienzo F, Collet C. 2014. The neurofunctional architecture of motor imagery. Funct Magn Reson Imaging 1:16.

Greer SM, Trujillo AJ, Glover GH, Knutson B. 2014. Control of nucleus accumbens activity with neurofeedback. Neuroimage 96:237-244.

Hétu S, Grégoire M, Saimpont A, Coll M-P, Eugène F, Michon P-E, Jackson PL. 2013. The neural network of motor imagery: an ALE meta-analysis. Neurosci Biobehav Rev 37: 930-949.

Jeannerod M. 1994. The representing brain: neural correlates of motor intention and imagery. Behav Brain Sci 17:187.

Koush Y, Rosa MJ, Robineau F, Heinen K, Rieger SW, Weiskopf N, et al. 2013. Connectivity based neurofeedback: dynamic causal modeling for real-time fMRI. Neuroimage 81 : 422-430.

Liew S-L, Rana M, Cornelsen S, Fortunato de Barros Filho M, Birbaumer N, Sitaram R, et al. 2016. Improving motor corticothalamic communication after stroke using real-time fMRI connectivity-based neurofeedback. Neurorehabil Neural Repair 30:671-675.

Linden DEJ, Turner DL. 2016. Real-time functional magnetic resonance imaging neurofeedback in motor neurorehabilitation. Curr Opin Neurol 29:412-418.

Liuzzi G, Hörniss V, Zimerman M, Gerloff C, Hummel FC. 2011. Coordination of uncoupled bimanual movements by strictly timed interhemispheric connectivity. J Neurosci 31: 9111-9117.

Mendes PA, Marinho DA, Petrica JD, Silveira P, Monteiro D, Cid L. 2016. Tradução E Validação Do Movement Imagery Questionnaire-3 (MIQ-3) Com Atletas Portugueses. Motricidade 12:149.

Mehler DMA, Williams AN, Krause F, Lührs M, Wise RG, Turner DL, et al. 2019. The BOLD response in primary motor cortex and supplementary motor area during kinesthetic motor imagery based graded fMRI neurofeedback. Neuroimage 184:36-44.

Minshew NJ, Keller TA. 2010. The nature of brain dysfunction in autism: functional brain imaging studies. Curr Opin Neurol 23:124-130.

New AB, Robin DA, Parkinson AL, Duffy JR, McNeil MR, Piguet O, et al. 2015. Altered resting-state network connectivity in stroke patients with and without apraxia of speech. Neuroimage Clin 8:429-439.

Piokenhain L. 1984. Chapter Vb Towards a holistic conception of movement control. Adv Psychol 17:505-528.

Poston KL, Eidelberg D. 2012. Functional brain networks and abnormal connectivity in the movement disorders. Neuroimage 62:2261-2270.

Rao SM, Bandettini PA, Binder JR, Bobholz JA, Hammeke TA, Stein EA, Hyde JS. 1996. Relationship between finger movement rate and functional magnetic resonance signal change in human primary motor cortex. J Cerebr Blood Flow Metabol 16:1250-1254.

Ridderinkhof KR, Van Den Wildenberg WP, Segalowitz SJ, Carter CS. 2004. Neurocognitive mechanisms of cognitive control: the role of prefrontal cortex in action selection, response inhibition, performance monitoring, and rewardbased learning. Brain Cognit 56:129-140.

Rota G, Sitaram R, Veit R, Erb M, Weiskopf N, Dogil G, Birbaumer N. 2009. Self-regulation of regional cortical activity using real-time fMRI: the right inferior frontal gyrus and linguistic processing. Human Brain Mapp 30:1605-1614. 
Ruiz S, Buyukturkoglu K, Rana M, Birbaumer N, Sitaram R. 2014. Real-time fMRI brain computer interfaces: selfregulation of single brain regions to networks. Biol Psychol 95:4-20.

Schubotz RI, Anwander A, Knösche TR, von Cramon DY, Tittgemeyer M. 2010. Anatomical and functional parcellation of the human lateral premotor cortex. Neuroimage 50:396-408.

Sitaram R, Lee S, Ruiz S, Birbaumer N. 2011. Real-time regulation and detection of brain states from fMRI signals. In: Coben R, Evans J (eds.) Neurofeedback and Neuromodulation Techniques and Applications. London, UK: Academic Press; p. 227.

Sitaram R, Ros T, Stoeckel L, Haller S, Scharnowski F, LewisPeacock J, et al. 2017. Closed-loop brain training: the science of neurofeedback. Nat Rev Neurosci 18:86-100.

Spetter MS, Malekshahi R, Birbaumer N, Lührs M, van der Veer $\mathrm{AH}$, Scheffler K, et al. 2017. Volitional regulation of brain responses to food stimuli in overweight and obese subjects: a real-time fMRI feedback study. Appetite 112:188-195.

Stephan KE, Baldeweg T, Friston KJ. 2006. Synaptic plasticity and dysconnection in schizophrenia. Biol Psychiatry 59: 929-939.

Sulzer J, Haller S, Scharnowski F, Weiskopf N, Birbaumer N, Blefari ML, et al. 2013. Real-time fMRI neurofeedback: progress and challenges. Neuroimage 76:386-399.

Talairach J, Tournoux P. 1988. Co-Planar Stereotaxic Atlas of the Human Brain: 3-Dimensional Proportional System: An Approach to Cerebral Imaging. New York, NY: Thieme Medical Publishers.

Thibault RT, MacPherson A, Lifshitz M, Roth RR, Raz A. 2018. Neurofeedback with fMRI: a critical systematic review. Neuroimage 172:786-807.

Weiskopf N. 2012. Real-time fMRI and its application to neurofeedback. Neuroimage 62:682-692.
Weiskopf N, Mathiak K, Bock SW, Scharnowski F, Veit R, Grodd W, et al. 2004. Principles of a brain-computer interface (BCI) based on real-time functional magnetic resonance imaging (fMRI). IEEE Trans Biomed Eng 51:966-970.

Weiskopf N, Scharnowski F, Veit R, Goebel R, Birbaumer N, Mathiak K. 2004. Self-regulation of local brain activity using real-time functional magnetic resonance imaging (fMRI). J Physiol Paris 98:357-373.

Xie F, Xu L, Long Z, Yao L, Wu X. 2015. Functional connectivity alteration after real-time fMRI motor imagery training through self-regulation of activities of the right premotor cortex. BMC Neurosci 16:1.

Zhang H, Tian J, Zhen Z. 2007. Direct measure of local region functional connectivity by multivariate correlation technique. Conf Proc IEEE Eng Med Biol Soc 2007:5231-5234.

Zilverstand A, Sorger B, Zimmermann J, Kaas A, Goebel R. 2014. Windowed correlation: a suitable tool for providing dynamic fMRI-based functional connectivity neurofeedback on task difficulty. PLoS One 9:e85929.

Zotev V, Phillips R, Young KD, Drevets WC, Bodurka J. 2013. Prefrontal control of the amygdala during real-time fMRI neurofeedback training of emotion regulation. PLoS One 8: e79184.

Address correspondence to: Miguel Castelo-Branco CIBIT, Coimbra Institute for Biomedical Imaging and Translational Research Institute of Nuclear Sciences Applied to Health (ICNAS) University of Coimbra

Coimbra 3000-548

Portugal

E-mail: mcbranco@fmed.uc.pt 